Revue des patrimoines

39 | 2019

Imagerie numérique et patrimoine culturel : enjeux scientifiques et opérationnels

\title{
Imagerie numérique et représentation des données en archéologie
}

Digital imagery and the representation of archaeological information

Sylvie Eusèbe

\section{(2) OpenEdition}

\section{Journals}

Édition électronique

URL : https://journals.openedition.org/insitu/21467

DOI : $10.4000 /$ insitu. 21467

ISSN : 1630-7305

Éditeur

Ministère de la Culture

Référence électronique

Sylvie Eusèbe, « Imagerie numérique et représentation des données en archéologie », In Situ [En ligne], 39 | 2019, mis en ligne le 24 mai 2019, consulté le 21 septembre 2021. URL : http:// journals.openedition.org/insitu/21467; DOI : https://doi.org/10.4000/insitu.21467

Ce document a été généré automatiquement le 21 septembre 2021.

\section{cc) $(1) \ominus$}

In Situ Revues des patrimoines est mis à disposition selon les termes de la licence Creative Commons Attribution - Pas d'Utilisation Commerciale - Pas de Modification 4.0 International. 


\title{
Imagerie numérique et représentation des données en archéologie
}

Digital imagery and the representation of archaeological information

\author{
Sylvie Eusèbe
}

\section{Introduction}

1 L'évolution de la représentation en archéologie s'apparente, pour une grande part, à la continuelle recherche de moyens permettant une transcription la plus fidèle possible de l'objet original en volume sur un support plan. Les vues en géométral se distinguent progressivement de la perspective, les conventions et les règles de représentation se construisent peu à peu, et la complémentarité du plan, de la coupe et de l'élévation s'affirme. Le rendu graphique rend compte de l'observation de plus en plus précise, s'éloignant du dessin artistique pour adopter la rigueur du tracé technique. Et enfin, l'habillage de la figure s'enrichit progressivement pour répondre aux exigences scientifiques croissantes.

2 Après une rapide évocation de l'évolution de la représentation des vestiges en archéologie, nous aborderons les changements observés actuellement, à travers des exemples tirés de la production de l'Inrap. En effet, que ce soit par le biais de technologies captant le réel en trois dimensions, de logiciels de traitement de données ou encore du dessin assisté par ordinateur, le numérique entraîne de nombreux changements dans la représentation des phénomènes et objets archéologiques, et on parle désormais d'imagerie numérique pour désigner les images produites par ces procédés. 


\section{Les vues en géométral et en perspective}

3 Avant la Renaissance, la représentation en plan, coupe et élévation ne fait pas de doute pour la conception de grands édifices (religieux notamment), mais il existe des systèmes de représentation à base de multiples rabattements que nous avons aujourd'hui bien du mal à «redresser» pour retrouver l'objet en volume. Par exemple, les dessins de machines du cahier de Villard de Honnecourt ${ }^{1}$ (première moitié du XIII ${ }^{e}$ siècle) sont loin d'être d'une lecture évidente ${ }^{2}$, alors que celle de ses plans, élévations et même vues perspectives de bâtiments, nous est encore familière.

Ce sont des architectes et des artistes qui, au début du $\mathrm{Xv}^{\mathrm{e}}$ siècle, théorisant et diffusant les règles sur les projections et la perspective centrale, rendent possible le report des grandeurs, des proportions et de ce qui caractérise l'objet à représenter. Ils ébauchent en outre la démarche de l'archéologue, en fouillant, mesurant et dessinant les vestiges de Rome, en plan et élévation, pour en comprendre la construction. Théorie, technique et soif de connaissances sur ce qui a fait la grandeur de la civilisation antique semblent intimement imbriquées.

5 En France, l'ouvrage du juriste et humaniste Jean Poldo d'Albenas intitulé Discours historial de l'antique et illustre cité de Nismes, publié en 1559-1560, est le premier à présenter un plan orienté avec une échelle ${ }^{3}{ }^{4}$. Les représentations des vestiges antiques présentent différentes vues générales et de détail, en plan, coupe, perspective à un point de fuite, et vue partiellement écorchée. Les dessins sont accompagnés de cotes, d'échelle en toises et en pieds, de trames (dans les parties coupées, sur les surfaces), et d'une nomenclature (lettres dans le dessin renvoyant à une description textuelle), c'est-à-dire les principaux éléments qui constituent encore l'habillage de la figure.

6 Cependant, comme aujourd'hui, la compréhension d'un objet à l'aide de ses vues en géométral que sont le plan, l'élévation et la coupe n'est pas intuitive, et on rencontre jusqu'au $\mathrm{XVIII}^{\mathrm{e}}$ siècle des représentations qui "mélangent" plan et élévation en présentant par exemple des façades rabattues le long de rues vues en plan. La représentation perspective, quant à elle, connaît de nombreux tâtonnements jusqu'à la fin $d u \mathrm{XVIII}^{\mathrm{e}}$ siècle, et bien que celle-ci soit utilisée par l'architecte graveur Jacques Androuet du Cerceau pour décrire les Antiquités romaines de Guillaume du Choul (1538), Valérie Auclair note qu' « un lecteur au milieu du Xvi ${ }^{\mathrm{e}}$ siècle n'est pas nécessairement apte à reconnaître un dessin en perspective, et donc à reconstruire la réalité optique qui est ainsi déformée $»^{5}$.

$7 \mathrm{Au}$ début du XVII ${ }^{\mathrm{e}}$ siècle, la seule description des antiquités n'apparaît plus suffisante, et on cherche à en expliquer les usages et les fonctions ${ }^{6}$. L'architecte Antoine Desgodets publie en 1682 Les Édifices antiques de Rome mesurés et dessinés très exactement. Fruit de ses propres fouilles et mesures, ces relevés utilisent les différentes vues que sont le plan, la coupe et l'élévation. Toutes les planches publiées présentent un rendu similaire, caractéristique du dessin d'architecture : le vestige est dessiné comme s'il était neuf ou encore à l'état de projet. Cependant, certains dessins mélangent ce rendu à celui de l'archéologie naissante ${ }^{7}$, et oscillent entre un rendu « artistique » (les blocs ruinés), et un rendu «technique» (les parties disparues restituées par leur contour en trait interrompu). 
8 Selon les compétences du dessinateur et les exigences du commanditaire, les dessins peuvent avoir des rendus variables, comme ce dessin de l'arc de triomphe de Langres ${ }^{8}$ qui figure dans le recueil de dessins Antiquités des Gaules du collectionneur Roger de Gaignières (1642-1715), à mi-chemin entre une représentation héritée de la Renaissance, voire plus ancienne, et celle qui commence à se répandre, symbolisée ici par l'échelle graphique qui renseigne sur la grandeur du vestige alors que la vue est une perspective à un point de fuite.

\section{Le rendu et l'habillage de la figure}

$9 \mathrm{Au} \mathrm{XVIII}^{\mathrm{e}}$ siècle, le dessin d'archéologie évolue vers des représentations mêlant certains aspects du rendu « réaliste » et du rendu « technique ».

Daté de 1760, le relevé de «La Pierre Levée », près de Poitiers, par l'ingénieur Duchesne, en est un bon exemple 9 . La mise en concordance des vues de face, de profil et de dessus de ce dolmen est typique du dessin des ingénieurs. Ce dessin mêle au rendu technique (les traits interrompus pour indiquer les blocs cachés par la dalle de couverture), un rendu plus artistique (cassures, végétation). Il est intéressant de noter que la publication de ce dessin, dans un des recueils du comte de Caylus ${ }^{10}$, donne lieu à une modification de la mise en page de la planche qui déconnecte les différentes vues entre elles, détruisant ainsi la logique initiale de leur agencement. Il est possible que les contraintes du format d'impression en soient la cause, mais il est également possible que la signification de la disposition des vues par Duchesne n'ait pas été perçue par le graveur.

11 Parallèlement, l'exigence scientifique se renforce, et emprunte à l'illustration naturaliste la manière de rendre compte graphiquement d'un réel minutieusement observé et que l'on s'attache à retranscrire, même si les techniques des professionnels des relevés ne sont pas maitrisées. C'est le cas des dessins du comédien Pierre Beaumesnil ${ }^{11}$ qui, malgré leurs maladresses, apportent de précieux renseignements archéologiques: assises, scellement et cassure des blocs, ou arases de briques ${ }^{12}$ sont représentés. Cet exemple incite à ne pas rejeter a priori les travaux des amateurs d'aujourd'hui qui ne seraient pas conformes aux standards de notre époque.

Le début du XIX ${ }^{e}$ siècle est marqué par la publication des travaux de l'expédition d'Égypte, dont les auteurs ont reçu l'enseignement des grandes écoles qui forment les dessinateurs, les ingénieurs et les architectes, puis ces derniers dominent le dessin archéologique, notamment à l'occasion des envois de Rome. Ceux-ci, par l'extrême soin apporté à rendre le réel le plus «réel possible » par l'usage de la couleur et de l'ombrage, préfigurent certaines des images numériques d'aujourd'hui. L'art que déploie l'architecte Félix Duban pour rendre compte de la texture des parements, de l'usure des blocs et du volume du vestige, tout en traçant une vue en géométral ${ }^{13}$, fait place à la maîtrise technique de la modélisation 3D dont est issue cette orthophotographie de la coupe d'un massif de maçonnerie (fig. 1). 
Figure 1
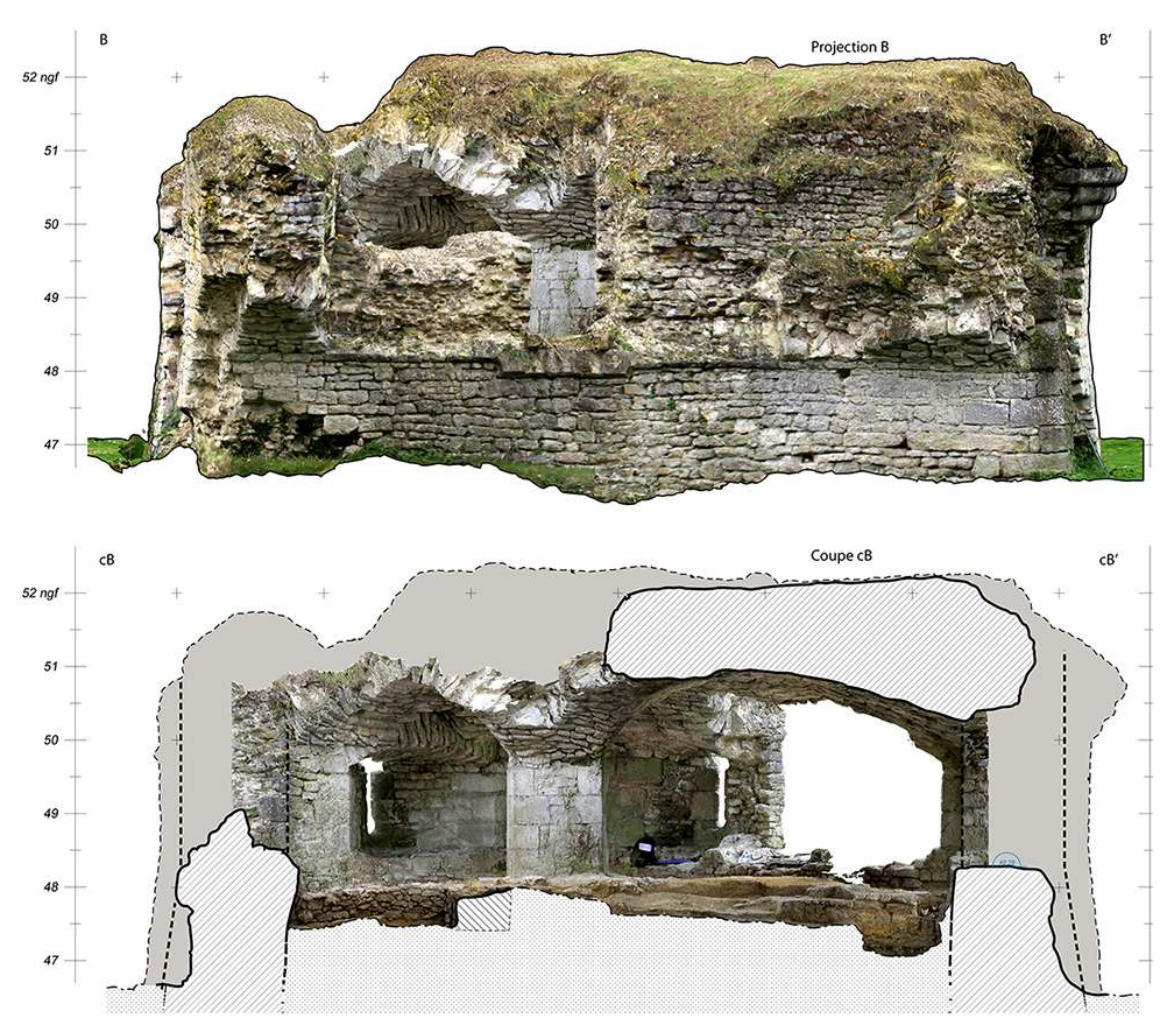

Orthophotographie, coupe longitudinale dans le ravelin du château de Beynes (Yvelines). F. Duban laisse en blanc les parties coupées et détaille les arrachements; ici les maçonneries coupées sont hachurées et les arrachements grisés.

(c) P. RAYMOND, L. DECOCK, 2016, INRAP.

$13 \mathrm{Au} \mathrm{Xx}^{\mathrm{e}}$ siècle, les champs d'étude de l'archéologie se multiplient et de nouvelles méthodes voient le jour (prospection, fouille en aire ouverte, enregistrement alliant la description textuelle, le dessin et la photographie). Les vestiges perçus sont de plus en plus ténus (fosse, fossé, trou de poteau, niveau d'occupation), leur représentation s'approprie des symboles venant de l'architecture et de la cartographie, invente une nomenclature spécifique à la numérotation des vestiges et des strates, recourt à l'usage de traits continus, interrompus et mixtes avec différentes épaisseurs. L'orientation et l'échelle sont systématiquement indiquées, tous les symboles sont légendés, des descriptions textuelles sont jointes au dessin. En plan comme en coupe, on s'astreint à localiser le vestige dessiné à l'aide de coordonnées géographiques et d'altitudes formulées dans les systèmes de référence légaux.

\section{Une représentation « hybride »}

Fin $\mathrm{xx}^{\mathrm{e}}$-début $\mathrm{xxI}^{\mathrm{e}}$ siècle, la représentation archéologique bénéficie de plus en plus rapidement des avancées technologiques : par exemple, une quinzaine d'années a suffi pour que l'encrage traditionnel des minutes de terrain au rapidographe soit remplacé par la mise au net informatisée. La technologie numérique permet également de recourir plus facilement que par le passé à la photographie, notamment comme aide au relevé, en évitant la prise des mesures et leur report. Par ailleurs, la complémentarité du dessin et de la photographie est renforcée par leur « mélange » au sein d'une même illustration, ce 
qui est particulièrement enrichissant pour la représentation des objets (fig. 2). En effet, si le dessin au trait permet la comparaison avec les représentations plus anciennes et surtout, rend compréhensible ce qu'il fait voir en l'interprétant, il peine à rendre compte de l'aspect des surfaces et des couleurs, ce dont s'acquitte revanche la photographie.

Figure 2
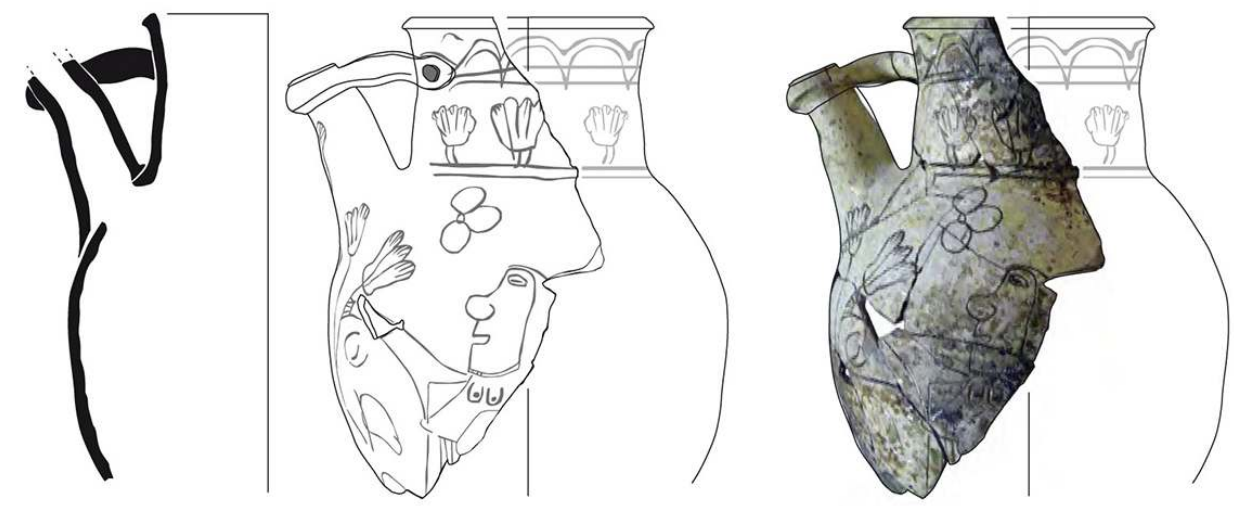

Représentation d'une céramique, photographie numérique et dessin vectoriel.

(c) E. Bayen, S. Bocquet, vers 2012, Inrap. lasergrammétrie, est en train de transformer l'aspect de leur enregistrement visuel. Pour les archéologues, les premières images issues d'une modélisation 3D sont produites par des cabinets de géomètres ou des entreprises spécialisées. Ce sont en général des impressions écran de la maquette numérique (donc une vue en perspective sans point de fuite) qui ne présentent pas l'habillage conventionnel (échelle et orientation en particulier). Ce n'est que lorsque les archéologues se rapprochent de ces producteurs d'images, ou qu'ils les produisent eux-mêmes, qu'elles incorporent peu à peu les codes des représentations antérieures.

Sur le terrain, la représentation des vestiges « s'hybride » également. La minute, ce relevé des vestiges en plan, coupe ou élévation, est depuis des décennies réalisée au crayon sur papier millimétré ou calque, à l'aide du minutieux report, à l'échelle choisie, d'une quantité de mesures. L'orthoimage issue du lever 3D modifie cette pratique : en effet, un lever 3D est réalisé dès qu'il est nécessaire de procéder à un relevé, et une orthoimage, en plan ou en coupe, est imprimée pour être annotée devant le vestige (fig. 3). Par ailleurs, les orthoimages ne sont pas systématiquement imprimées en couleur, certains détails (notamment ceux des maçonneries) ressortant mieux en noir et blanc. 


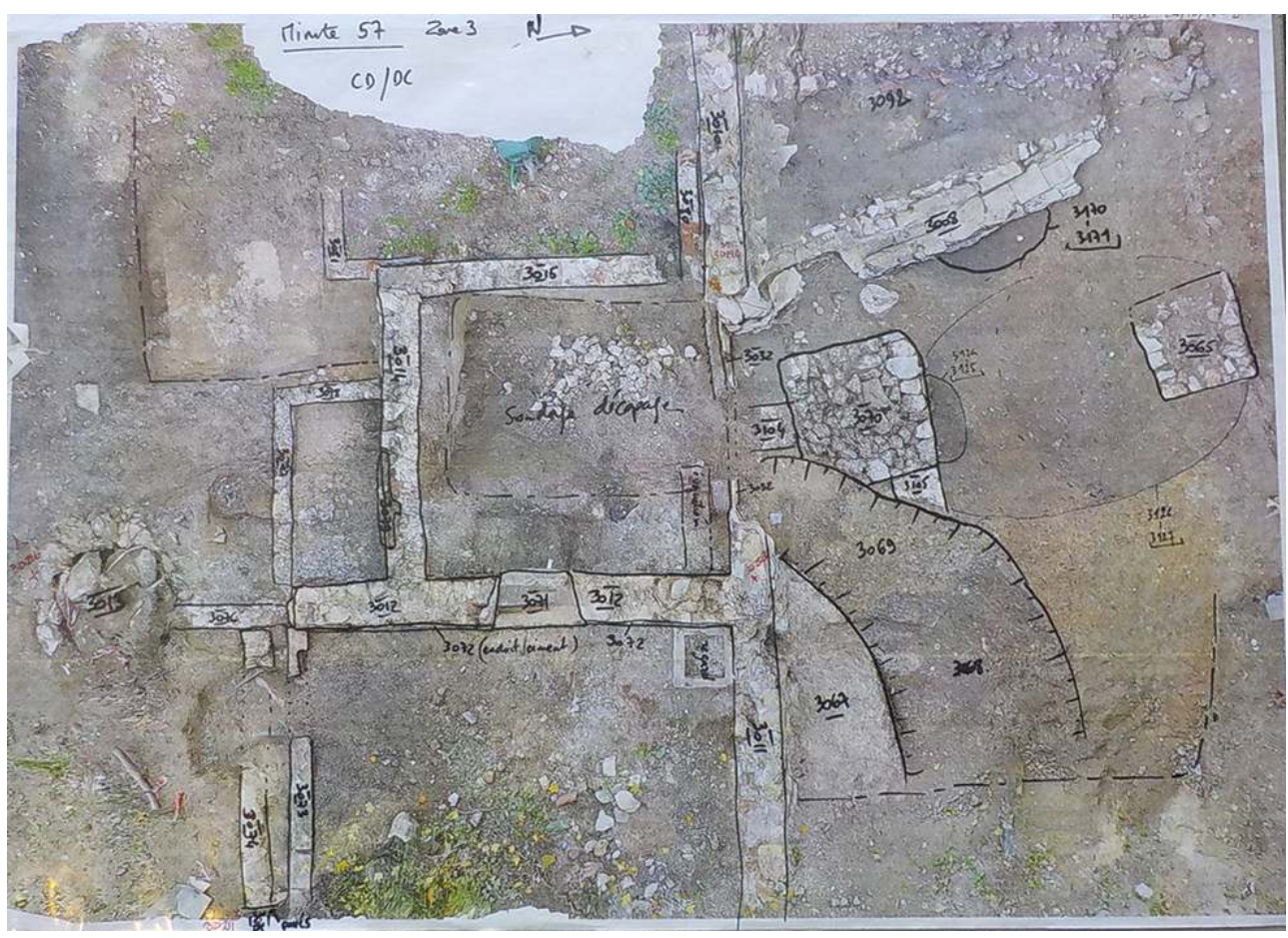

Orthoimage annotée tenant lieu de minute de terrain.

(c) R. El-Hajaoui, D. Couturier, 2016, Inrap.

On pourrait croire que ces images à l'aspect réaliste sont immédiatement compréhensibles grâce à leur analogie avec la réalité sensible. Cependant, arriver à lire les différents plans de l'orthoimage pour l'identifier au vestige que l'on a sous les yeux peut demander un temps d'adaptation. Le cerveau doit la traduire pour l'assimiler à la réalité, et la mettre en volume par le biais d'une image mentale, puisque c'est la machine qui projette un volume sur la surface plane de la minute, et non plus le dessinateur.

Pour être efficace, la mise en place de ce processus dans le cadre opérationnel nécessite une nouvelle organisation et des moyens informatiques nouveaux à proximité du chantier, mais le gain de temps en phase terrain semble réel. Cependant, il reste à expérimenter des méthodes de traitement de ces "nouvelles minutes », notamment pour réaliser efficacement les figures à publier dans le rapport d'opération ou tout autre support.

20 Ceci explique peut-être que, bien que le lever 3D produise une documentation variée, en 3D ou en 2D, numérique ou papier, il est encore rare de rencontrer des figures au rendu enrichi par ces techniques de lever automatiques.

21 Quelques exemples donnent cependant une idée de l'esprit créatif dans lequel de telles images voient le jour. Comme les photographies redressées avant elles, les orthoimages viennent accompagner le dessin qui reste nécessaire pour montrer l'interprétation de l'archéologue (fig. 4). 
Figure 4

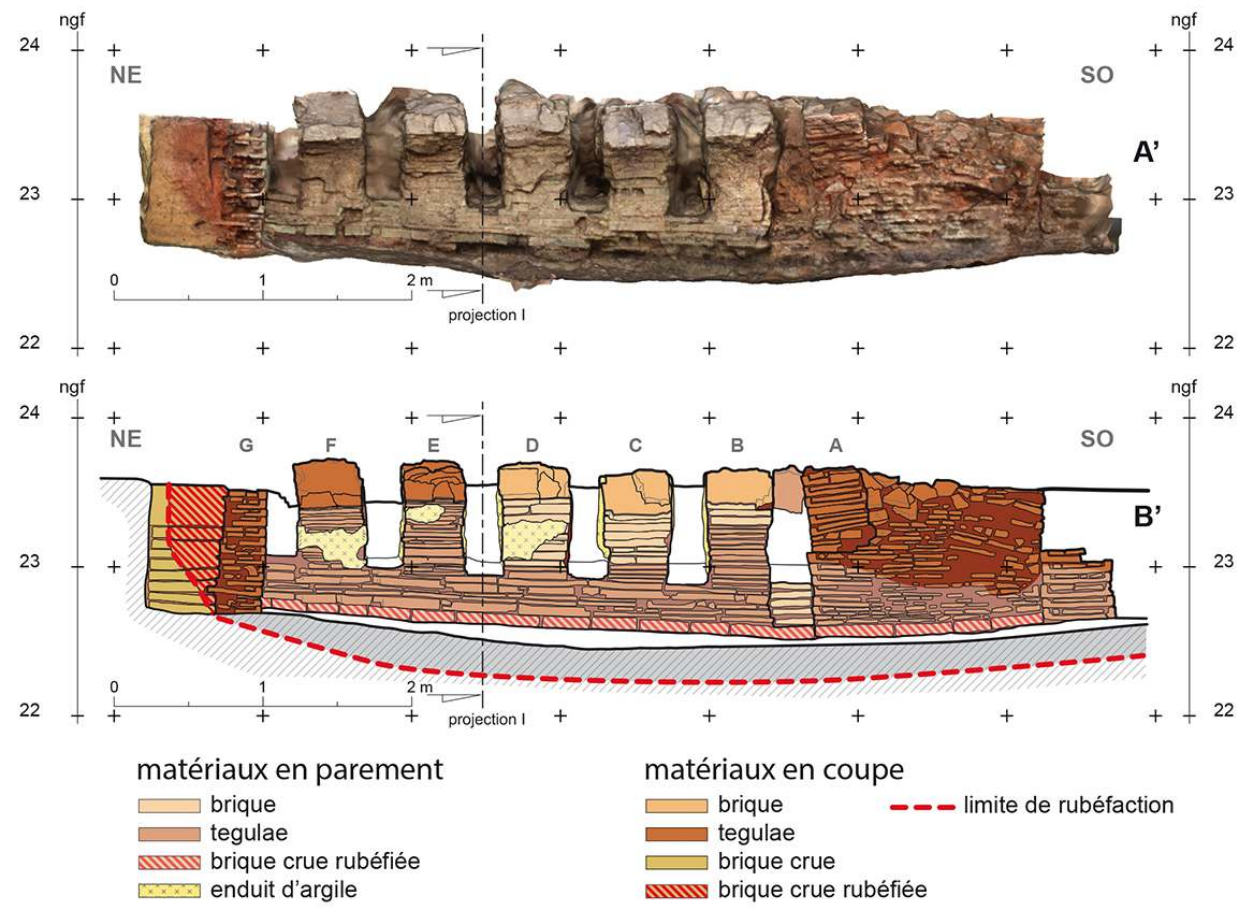

Orthophotographie de la coupe longitudinale d'un four et dessin interprétatif vectoriel réalisé sur le terrain à l'aide de l'orthophoto (Mours, Val-d'Oise).

(C) P. Raymond, A. Mondoloni, vers 2015, Inrap.

Des rendus nouveaux sont bien sûr testés (fig. 5), mais une évolution significative réside dans la juxtaposition d'une vue identique avec des rendus différents (fig. 6), parce qu'une seule ne peut supporter toutes les informations dont on dispose, et que l'on peut, et souhaite, transmettre. 
Figure 5

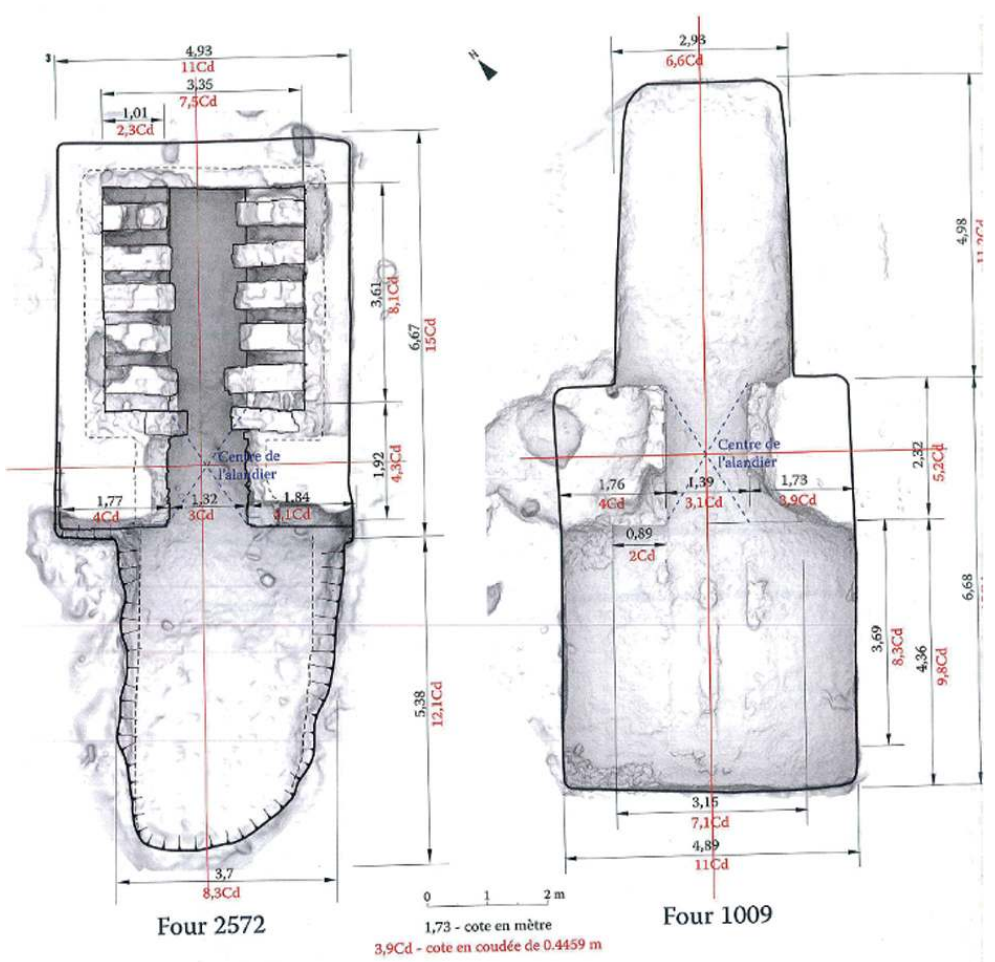

Plans de deux fours de tuilier (Mours, Val-d'Oise). Le grisé qui imite le crayon ne représente pas les ombres, mais participe néanmoins à la lecture du relief.

(c) P. Raymond, A. Mondoloni, vers 2015, Inrap. 


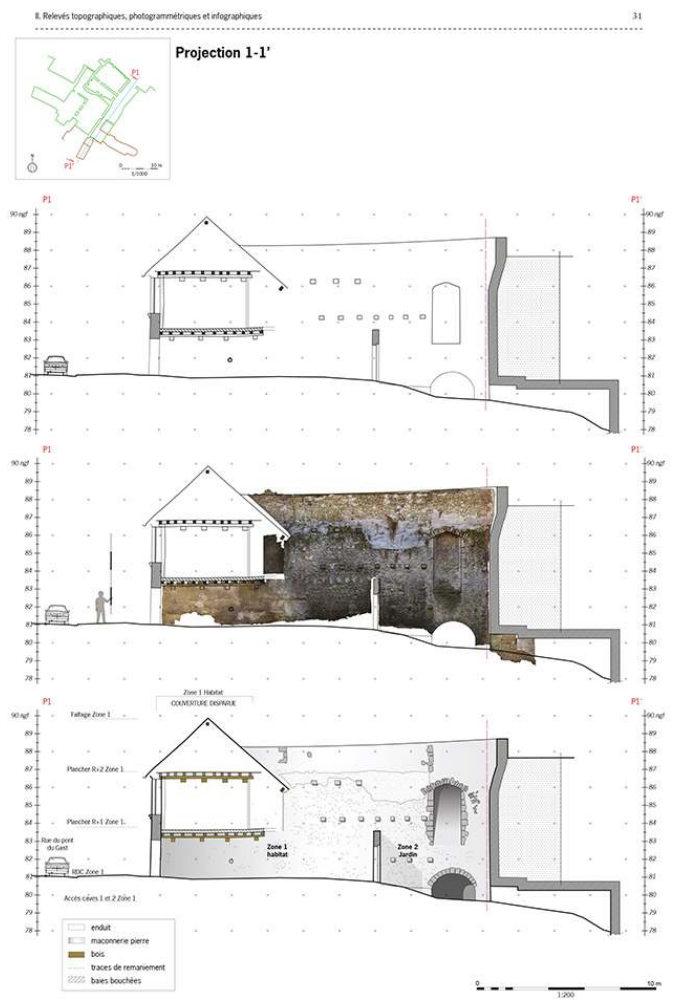

Planche d'un rapport d'opération, une même coupe longitudinale dans un bâtiment présentant trois rendus complémentaires.

(c) A. Cetout, 2016, Inrap.

Si les produits issus du modèle 3D sont de plus en plus utilisés, le modèle lui-même semble encore sous-exploité. Les difficultés techniques rencontrées par un grand nombre de chercheurs peu rompus à l'environnement informatique jouent, et principalement celles liées à la visualisation et la manipulation du modèle (viewer jugé encore peu ergonomique et aux fonctionnalités pas toujours adaptées aux besoins de l'archéologue).

24 Un autre frein à son usage est la modification de l'expression de la résolution : du 1 centimètre sur le papier pour $\mathrm{X}$ centimètres sur le terrain, on est passé à $\mathrm{X}$ centimètres par pixel ; le référentiel mental qui associait un « niveau de détail » à une échelle doit être recréé, un peu comme lors du passage du franc à l'euro.

Pourtant, la visualisation toujours plus complète et précise de l'objet réel (en matière de forme, de dimension, de volume, et de niveau de détail de ses composants) et les possibilités de calcul qui en découlent, attirent de plus en plus. Cependant, dans ce cas, le danger est de ne pas résister à l'exhaustivité pour elle-même, c'est-à-dire de modéliser sans objectifs techniques et scientifiques précis, et sans distinction, tous les vestiges rencontrés. Le risque de penser que nos représentations sont de plus en plus exhaustives, grâce à la quantité de données enregistrées, et objectives, parce que l'humain n'intervient pas directement dans leur captation, est également bien réel. Le recul nécessaire pour avoir un regard critique sur nos méthodes de travail peut alors être affecté.

Par ailleurs, dans un contexte soumis aux pressions économiques, le modèle 3D est présenté comme une opportunité pour réduire le temps passé sur le terrain. La tentation est alors forte d'effectuer de plus en plus d'observations sur la réplique numérique, au 
lieu de les réaliser sur l'objet réel. Pourtant, il est un peu tôt pour percevoir dans quelle mesure le modèle 3D peut devenir un support d'observation et un outil de compréhension au même titre qu'un relevé manuel ou l'annotation d'une orthoimage effectués en présence $d u$ vestige réel. Bien que les archéologues s'accordent sur la nécessité incontournable d'effectuer les observations sur le terrain, le recul manque encore pour cerner la teneur et l'ampleur d'un éventuel risque de perte d'informations si réaliser les observations sur le modèle numérique venait à se généraliser.

Et que pourrait être une archéologie dans laquelle la réalité serait uniquement appréhendée par le sens de la vue ? Où le vestige ne serait perçu que par son apparence, par son image? Même si la conscience que la chose réelle n'est pas là demeure, quelles modifications de la perception cela pourrait-il entrainer? «La prééminence de la vue, que tout renforce dans la civilisation moderne (le rôle de l'écrit, puis du dessin et du calcul, la reproduction et la diffusion industrielles des images), nous place devant le dilemme du "voyeur" : voir, est-ce ou non connaitre, posséder, avoir accès ? $»^{14}$

Si l'attirance pour le visuel se traduit dans le domaine de l'archéologie par le succès de l'imagerie photo-réaliste en 2D ou 3D, il existe parallèlement tout un pan de la représentation de la donnée archéologique qui tend vers l'abstraction, depuis notamment les diagrammes de William Playfair (1759-1823) ou Joseph Minard (1781-1870), et dont l'évolution en lien avec la micro-informatique serait également à analyser dans une perspective historique.

\section{L'évolution des conventions et règles de représentation}

Jusqu'à la fin du $\mathrm{xx}^{\mathrm{e}}$ siècle, le dessinateur en archéologie, doté de compétences innées pour le dessin, faisait son apprentissage pratique sur les chantiers, relevant manuellement les vestiges et mettant au net tout aussi manuellement ses minutes, en appliquant les conventions de représentation de la discipline transmises par ses pairs.

D'une manière générale, la démocratisation due à l'informatique tend à faire oublier aux utilisateurs que, dans tous les domaines, il existe des conventions et des règles justifiées qui ne peuvent être assimilées à la maîtrise de l'outil. Or, avec le développement de la micro-informatique, les logiciels permettent aux archéologues non spécialistes du dessin d'en réaliser.

31 De plus, l'énergie nécessaire pour maîtriser certains outils de DAO ou de $\mathrm{CAO}^{15}$ semble faire primer leur apprentissage sur celui des règles de représentation, qui s'appliquent pourtant quels que soient les outils utilisés. Aussi, le formidable potentiel de renouveau et d'évolution offert par l'imagerie numérique est-il tempéré par des rendus qui peuvent être jugés inappropriés, au regard des règles de représentation et de la sémiologie graphique bien sûr, mais surtout parce qu'ils nuisent à la compréhension de ce qui est représenté.

À titre d'exemple, le rendu de certains dessins assistés par ordinateur trahit un travail moindre sur les épaisseurs des traits par rapport aux dessins mis au net «à la main » à l'encre. Forte pour les premiers plans et les éléments participant à l'identification de l'objet représenté, moyenne pour les plans intermédiaires, et fine pour les arrière-plans et les détails jugés de moindre importance, l'épaisseur des contours est pourtant un moyen efficace de rendre plus immédiatement intelligibles le volume de l'objet et le 
discours proposé au lecteur. En outre, cette hiérarchisation visuelle des contours participe à la compréhension de l'objet au moment même de la réalisation du dessin.

L'habillage de la figure, pour sa part, voit certains de ses éléments, pourtant indissociables de la représentation scientifique depuis le xix siècle (ou même depuis certains dessinateurs de la Renaissance comme montré plus haut) disparaitre parce que désormais considérés inutiles. Le dessin assisté par ordinateur offre une rapidité d'exécution sans précédent, notamment parce qu'il réutilise les mêmes figurés, dessinés une fois pour toutes, et stockés dans une bibliothèque de symboles. Cependant, une échelle graphique, et même une flèche indiquant le nord, peuvent être supprimées au prétexte que le plan présente le carroyage Lambert, dont les valeurs des abscisses et des ordonnées donnent l'échelle et l'orientation. Pourtant, loin d'être redondantes, ces informations, en se recoupant entre elles, permettent de s'assurer de leur justesse, et participent ainsi à la qualité de la représentation.

Quant à l'utilisation de la vue en géométral, en plan comme en coupe, elle ne sera probablement pas délaissée tant que la diffusion des observations et des résultats archéologiques se fera majoritairement en 2D. Cependant, l'usage de plus en plus courant de la visualisation en 3D peut accroître le nombre d'archéologues rencontrant des difficultés à reconstruire mentalement le volume d'un objet à partir de ses différentes vues en 2D.

En même temps que ces changements dans la représentation des vestiges, la visualisation des données est également modifiée. En effet, les règles de la sémiologie graphique telles que les a posées Jacques Bertin dans les années $1960^{16}$ évoluent : d'une part les possibilités de traitement de la donnée par l'ordinateur se diversifient, et d'autre part les logiciels de traitement des données permettent de nouveaux rendus. De plus, de nouvelles variables visuelles adaptées à l'écran sont créées (par exemple : variation de l'intensité lumineuse, vitesse de déplacement ou rythme de clignotement d'un point). Cependant, la sémiologie graphique reste mal connue d'un grand nombre d'archéologues, et beaucoup n'ont pas toujours le réflexe de s'interroger sur la nature de leurs données pour en déduire leur représentation. En outre, les sites en ligne qui aident à choisir les gammes de couleurs ou les propositions faites par les logiciels (SIG notamment) ne sont guère pédagogues en la matière en permettant de mauvaises utilisations des variables.

L'erreur la plus fréquente consiste à utiliser la variable couleur pour exprimer une donnée quantitative relative ordonnée, au lieu de la variable valeur. S'écarter sciemment de la règle peut avoir un sens, mais s'en écarter sans savoir qu'il y a une règle amène à des représentations qui manquent leur but: elles ne traduisent pas visuellement l'évolution du phénomène (fig. 7, fig. 8). Une évolution pertinente, car elle permet la visualisation de la variation de données quantitatives, est l'utilisation conjointe des variables couleur et valeur. Elle a désormais sa place dans les ouvrages de cartographie, par exemple sous le terme de "gradation harmonique $»^{17}$. En définitive, une "sémiologie infographique " appliquée à l'archéologie reste à construire, à diffuser, et à faire évoluer de manière raisonnée. 
Figure 7

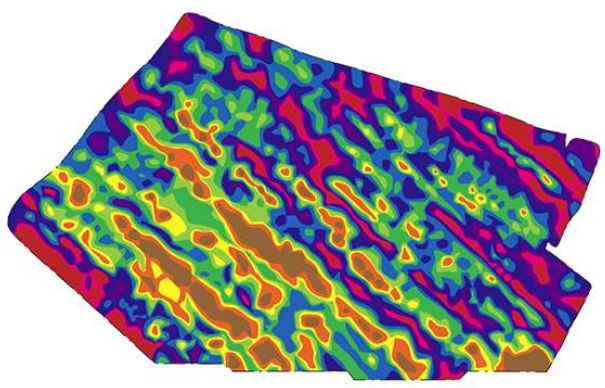

a $\square \square \square \square \square \square \square \square \square$

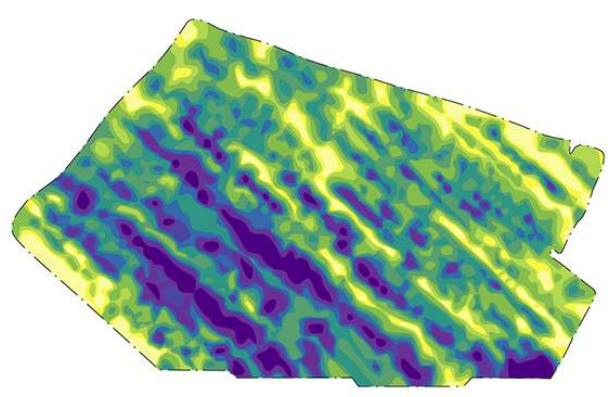

C

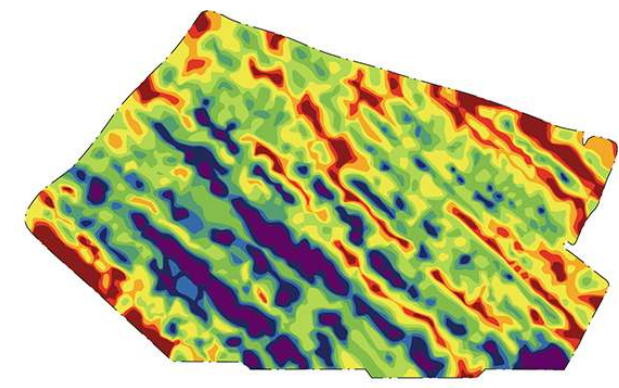

b

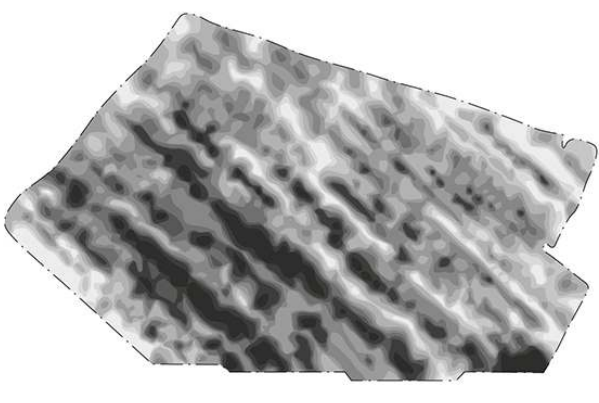

d $\square \square \square \square \square \square \square \square \square$

Plan fictif de l'intérieur d'un enclos fossoyé pour tester la représentation d'une donnée quantitative suivant ou non les principes de la sémiologie graphique. La variation, par exemple, d'un taux ou de l'altimétrie, n'est pas compréhensible sur les figures a et b car l'utilisation de la variable couleur n'est pas appropriée pour visualiser l'ordre (du plus faible au plus fort, du plus haut au plus bas). En revanche sur les figures c (variable couleur et valeur) et d (variable valeur) le phénomène est visible. (C) S. Eusèbe, 2018, Inrap. 

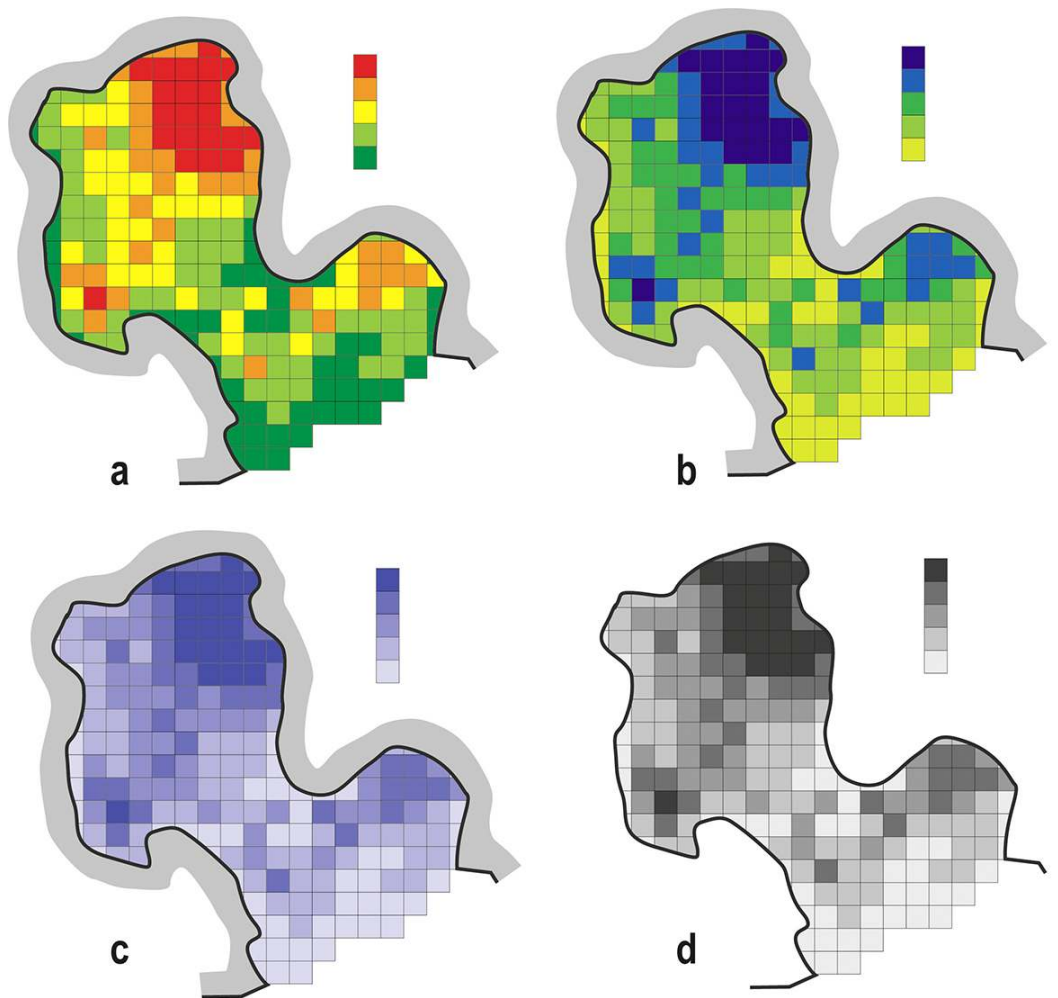

Plan fictif d'une grotte pour tester la représentation d'une donnée quantitative relative, par exemple une densité (nombre de restes par mètre carré). La figure a utilise la variable couleur déconseillée pour représenter ce type de donnée, la figure b à l'aide des variables couleur et valeur permet de voir l'évolution de la densité, mais les figures c et d, en n'utilisant que la variable valeur, sont plus explicites.

(C) S. Eusèbe, 2018, Inrap.

Les suppressions (échelle graphique et ses graduations, flèche du nord) ou les simplifications (épaisseur identique des traits) peuvent passer pour des détails. Critiquer une variable visuelle mal choisie peut passer pour du pédantisme. Mais une représentation archéologique efficace est composée de détails accumulés depuis plusieurs siècles pour répondre aux exigences scientifiques. Laisser certains d'entre eux disparaître, c'est risquer de perdre une partie du sens de ce qui est représenté (souvenons-nous des dessins de Villard de Honnecourt).

La traduction graphique d'une information répond au fonctionnement de nos systèmes perceptifs. Permettre certaines modifications en dehors de toute théorie, c'est non seulement risquer de n'être pas crédible aujourd'hui, mais aussi de ne plus être compris dans l'avenir.

Il apparait donc essentiel de transmettre les règles en même temps que les outils de représentation, de sensibiliser les différents acteurs, pour les uns, aux possibilités et limites techniques, pour les autres, aux conventions et règles de représentation en archéologie. 


\section{Appropriation, interprétation, transmission}

Et dans un avenir proche, quel chemin pourrait emprunter la représentation en archéologie?

Tout d'abord, il conviendrait que les archéologues s'approprient plus complètement le modèle 3D comme outil d'enregistrement, puis pour créer de véritables «figures en 3D " qui permettent de rendre compte des observations faites sur le terrain, ainsi que des interprétations et hypothèses proposées, comme on le fait avec les illustrations classiques. Les supports de diffusion, les outils logiciels, la formation des archéologues et les mentalités doivent encore évoluer pour rendre cela possible. Mais une meilleure connaissance des services que peuvent rendre l'imagerie numérique et la 3D permet déjà à certains archéologues de distinguer de nouveaux objectifs scientifiques. La tomodensitométrie par exemple, promet, en montrant l'invisible, d'être une source de découvertes scientifiques et d'innovations visuelles. Cependant, il faudra désormais être capable de s'adapter en permanence aux évolutions technologiques, et surtout logicielles.

Parallèlement, il ne faudrait pas céder à la facilité en s'en remettant à la représentation automatique proposée par les machines, se laisser ainsi « déresponsabiliser », et renoncer à l'interprétation de ces images sous prétexte que leur aspect photo-réaliste les rend intelligibles par tous. «La donnée n'est pas la pensée » comme titrait récemment une rencontre interdisciplinaire ${ }^{18}$; la donnée n'est pas le savoir.

43 Le prolongement logique de cette réflexion est la réaffirmation de la responsabilité de l'archéologue dans le tri et le choix des données et des images numériques qui doivent être transmises aux générations futures. C'est à lui de leur donner de la valeur en leur donnant une durée de vie, et de s'assurer qu'elles ne tomberont pas dans «le trou noir numérique ». Seuls l'accessibilité, le partage, et donc la gestion de données documentées (qui les a créées, pour quoi faire, comment, etc.) peuvent donner un tel gage de longévité.

4 Les nouvelles technologies invitent à reposer la question de la finalité de la représentation en archéologie. Ses buts sont multiples (description, étude, diffusion, conservation, etc.), ses techniques de réalisation et ses auteurs également. Ce n'est qu'en réaffirmant les règles de l'art de l'archéologie, en décrivant le cheminement intellectuel suivi, ses objectifs, ses limites, ses méthodes, ses degrés de certitude et ses sources que l'on conférera à ces nouvelles images la qualité scientifique qu'elles méritent.

\section{BIBLIOGRAPHIE}

AUCLAIR, Valérie. Dessiner à la Renaissance, la copie et la perspective comme instruments de l'invention. Rennes : PUR, 2010.

CAYLUS, Anne Claude de. Recueil d'antiquités égyptiennes, étrusques, grecques, romaines et gauloises. 7 volumes. Paris : 1752-1767. 
DESGODETS, Antoine. Les Édifices antiques de Rome mesurés et dessinés très exactement. Paris : J.-

B. Coignard, 1682 .

DU CHOUL, Guillaume. Antiquités romaines. Premier livre manuscrit, 1538.

EUSÈBE, Sylvie. « L'évolution de la représentation scientifique en archéologie ». Dans HOLIN, Alexandre (dir.). Espaces dessinés, espace du dessin. Actes du colloque « Dessiner-Tracer » à Villeneuve-d'Ascq/musée d'Art moderne en 2011. Villeneuve-d'Ascq : Presses universitaires du Septentrion, 2014

GALINIÉ, Henri. Tours antique et médiéval, lieux de vie, temps de la ville. Tours : FERACF, 2007.

LEMERLE, Frédérique. La Renaissance et les antiquités de la Gaule, l'architecture gallo-romaine vue par les architectes, antiquaires et voyageurs des guerres d'Italie à la Fronde. Turnhout : Brepols, 2005.

MONTFAUCON, Bernard de. L'Antiquité expliquée et représentée en figures. 15 volumes, Paris : Delaulne/Foucault/Clousier, 1719-1724.

POLDO D'ALBENAS, Jean. Discours historial de l'antique et illustre cité de Nîmes. Lyon : G. Rouille, 1559, 1560.

SCHNAPP, Alain. La Conquête du passé : aux origines de l'archéologie. Paris : Éditions Carré, 1993, Livre de Poche, 1998.

WIRTH, Jean. Villard de Honnecourt, architecte du XIII siècle. Genève : Librairie Droz, 2015.

Revues :

Études digitales. 2016-2, nº 2, "Le gouvernement des données".

Les Nouvelles de l'archéologie, 2016, n 146, "La 3D en archéologie"

http://journals.openedition.org/nda/ [consulté le 25/04/2019]

\section{NOTES}

1. - Voir par exemple le folio $44 \mathrm{du}$ cahier de Villard de Honnecourt: http://classes.bnf.fr/ villard/feuillet/index.htm [consulté le 25/04/2019].

2. - WIRTH, Jean. Villard de Honnecourt, architecte du XIII siècle. Genève : Librairie Droz, 2015.

3. - LEMERLE, Frédérique. La Renaissance et les antiquités de la Gaule : l'architecture gallo-romaine vue par les architectes, antiquaires et voyageurs, des guerres d'Italie à la Fronde. Turnhout : Brepols, 2005.

4. - Figure du circuit des murs antiques de Nismes, tiré de POLDO D’ALBENAS, Jean. Discours historial de l'antique et illustre cité de Nismes. Lyon : G. Rouille, 1559, 1560. Voir le site : http://bibliothequenumerique.inha.fr/viewer/11941/?offset=1\#page=42\&viewer=picture [consulté le 25/04/2019].

5. - AUCLAIR, Valérie. Dessiner à la Renaissance, la copie et la perspective comme instruments de l'invention. Rennes : PUR, 2010, p. 195.

6. - SCHNAPP, Alain. La Conquête du passé : aux origines de l'archéologie [1993]. Paris : Livre de Poche, 1998, p. 217-218.

7. - Chapiteau ionique et son entablement. Dans DESGODETZ, Antoine. Les Édifices antiques de Rome dessinés et mesurés très exactement. Paris : Jean-Baptiste Coignard, 1682. Voir le site: http:// bibliotheque-numerique.inha.fr/viewer/6987/?offset=1\#page=297\&viewer=picture [consulté le 25/04/2019].

8. - Dessin de l'arc de triomphe de Langres, tiré de GAIGNIÈRES, Roger de. Antiquités des Gaules. Voir le site : http://gallica.bnf.fr/ark:/12148/btv1b69376280 [consulté le 25/04/2019].

9. - SCHNAPP, Alain. La Conquête du passé... Op. cit., note 6, p. 294. 
10. - Gravure d'une pierre levée près de Poitiers, dans CAYLUS, Anne Claude de. Recueils d'antiquités égyptiennes, étrusques, grecques, romaines et gauloises. Paris : 1752-1767, vol. 4, voir le site : $\quad$ http://caylus-recueil.huma-num.fr/base/index.php?r=recueil/oeuvre/ detail\&id=1654\&indice-planche=1\&indice-photo=0 [consulté le 25/04/2019].

11. - Dessin d'une tour, tiré de BEAUMESNIL, Pierre. Carnet de notes et de dessins sur les Antiquités des villes de Saintes, Périgueux, Bénac, Guéret, etc. Voir le site: http://gallica.bnf.fr/ark:/12148/ btv1b10546954m/f72.item.zoom [consulté le 25/04/2019].

12. - GALINIÉ, Henri (dir), BORDEAUX, Patrick, SEIGNE, Jacques. Tours antique et médiéval, lieux de vie, temps de la ville. Tours : FERACF, 2007, p. 250-251.

13. - Album de dessins d'architecture réalisés par Félix Duban pendant son séjour à la villa Médicis, entre 1823 et 1828. T. 3 : Ostie, coupe du temple de Jupiter. Voir le site: http:// bibliotheque-numerique.inha.fr/viewer/7158/?offset=3\#page=108\&viewer=picture [consulté le 25/04/2019].

14. - THIBAUD, Paul. «Image première ». Dans MOURIER, Maurice (dir.). Comment vivre avec l'image. Paris : PUF, 1989, p. 44. Voir le site : http://gallica.bnf.fr/ark:/12148/bpt6k48058498/f29.image [consulté le 25/04/2019].

15. - Dessin assisté par ordinateur, conception assistée par ordinateur.

16. - BERTIN, Jacques. Sémiologie graphique. Les diagrammes. Les réseaux. Les cartes. Paris/La Haye : Mouton/Gauthier-Villars, 1967.

17. - LAMBERT, Nicolas, ZANIN, Christine. Manuel de cartographie. Principes, méthodes, applications. Paris : Armand Colin, 2016, p. 98-99.

18. - Voir le site: http://obvil.paris-sorbonne.fr/actualite/la-donnee-nest-pas-la-pensee/ jeu-14122017-0000 [consulté le 25/04/2019].

\section{RÉSUMÉS}

Depuis la Renaissance, la représentation des vestiges en archéologie a été influencée par la discipline d'origine des dessinateurs, et enrichie d'éléments au gré de l'évolution des sciences et des techniques. Il en va de même actuellement : la façon dont sont captées les données, et dont est produite l'image numérique qui les représente, a un impact tout aussi visible sur l'image archéologique. Nous sommes confrontés à des outils d'investigation qui rendent visible ce qui ne l'était pas : inventer une façon de représenter ce qui est inédit nous oblige à être créatifs. Nous avons à notre disposition des logiciels de traitement des données proposant des rendus graphiques nouveaux ou difficilement réalisables par le passé, on les expérimente donc. Le formidable potentiel de renouveau et d'évolution offert par l'imagerie numérique est cependant tempéré, comme auparavant, par des rendus graphiques qui peuvent être jugés inappropriés. Conséquence de la méconnaissance des règles de représentation ou de la technique utilisée, ou évolution bénéfique à la science? Des éléments, pourtant indissociables de la représentation scientifique depuis des décennies, disparaissent parce qu'ils sont désormais considérés comme inutiles ou redondants : peut-on imaginer les conséquences de ces disparitions à long terme ? À travers l'expérience issue de travaux menés à l'Inrap, on tâchera de cerner les usages de l'imagerie numérique qui se dessinent, et quels changements sont apportés dans la représentation imagée des données archéologiques. 
Since the Renaissance, the way archaeological remains have been represented has been based first of all on the draftsman's talents, later enriched according to scientific and technical progress. This is still true today. The way data is captured and the way digital images are produced have an equally visible impact on archaeological images. But our investigative tools can today render visible what was not visible before, and the representation of newness requires creativity. Our software can offer graphical renderings which are completely new or were difficult to produce in the past, so we try them out. But this tremendous potential for renewal and evolution that digital imaging offers must provide appropriate graphic renderings. Is this a question of a lack of knowledge about the rules of representation and the techniques used, or is it a beneficial progress for science? Some elements hitherto inseparable from scientific representation are disappearing today because they are now considered useless or redundant. But can we imagine the consequences of these losses in the long term? Through the experience of the work carried by the INRAP, France's national institution for preventive archaeologicy, we will try here to identify the emerging uses of digital imaging, and the way the representations of archaeological data are changing.

\section{INDEX}

Keywords : preventive archaeology, drawing, scientific imagery, 3D model, modelling, digital, orthoimage, survey, representation, technology, archaeological remains

Mots-clés : archéologie préventive, dessin, imagerie scientifique, modèle 3D, modélisation, numérique, orthoimage, relevé, représentation, technologie, vestiges archéologiques

\section{AUTEUR}

\section{SYLVIE EUSĖBE}

Ingénieur de recherche chargée de l'image, du dessin et de l'infographie à la Direction scientifique et technique, Inrap sylvie.eusebe@inrap.fr 